\title{
Systematic Approach to Microstructure Design of Ni-Base Alloys Using Classical Nucleation and Growth Relations Coupled with Phase Field Modeling
}

\author{
BILLIE WANG, Y.H. WEN, JEFF SIMMONS, and YUNZHI WANG
}

To analyze the formation of bimodal particle size distributions during precipitation, the dynamic competition for supersaturation by growth of existing precipitates and nucleation of new particles was studied under continuous cooling conditions with constant cooling rates. The nucleation rate was calculated according to classical nucleation theory as a function of local supersaturation and temperature. The depletion of matrix supersaturation by growth of existing particles was calculated from fully diffusion-controlled precipitate growth in an infinite matrix. Phase field simulations of $\gamma^{\prime}$ precipitation in a binary Ni-Al alloy were performed under continuous cooling conditions. Then the average and maximum matrix supersaturations were calculated and plotted onto the contours of nucleation rate and growth rate in concentration and temperature space. These methods were used iteratively to identify the window for bimodal particle size distributions.

DOI: $10.1007 / \mathrm{s} 11661-007-9405-1$

(c) The Minerals, Metals \& Materials Society and ASM International 2008

\section{INTRODUCTION}

THE advance of physics-based modeling allows microstructure design through processing to produce desired property blends. In Ni-base superalloys, for example, the bimodal particle size distribution (PSD) is believed to have a beneficial set of properties. Recent studies $^{[1]}$ have shown that deformation response of Ni-base disk alloys is governed by the detailed microstructural features of the bimodal $\gamma^{\prime}$ microstructure. These features include the average particle sizes, size distributions, and volume fractions of the secondary (large) and tertiary (small) $\gamma^{\prime}$ precipitates. A typical example of the bimodal microstructure observed in the disk alloys is shown in Figure 1. Optimizing the creep response of these alloys requires a close control of the microstructural features of both the secondary and tertiary particles as a function of alloy composition and heat treatment schedule.

Traditionally, controlling microstructural features has been an empirical effort, which is becoming both too costly and too slow for modern engine development cycles. Physics-based modeling promises to give a much tighter control of the microstructure and to make the

BILLIE WANG, GK-12 Fellow and YUNZHI WANG, Professor, are with the Department of Material Science and Engineering, The Ohio State University, Columbus, OH, 43210. Y.H. WEN, Research Scientist, is with UES, Inc., Dayton, OH 45432. JEFF SIMMONS, Research Scientist, is with the Air Force Research Laboratory, AFRL, MLLM, Wright Patterson AFB, Dayton, OH 45433. Contact e-mail: jeff.simmons@wpafb.af.mil

This article is based on a presentation given in the symposium entitled "Solid-State Nucleation and Critical Nuclei during First Order Diffusional Phase Transformations" which occurred October 15-19, 2006 during the MS\&T meeting in Cincinnati, OH, under the auspices of the TMS/ASMI Phase Transformations Committee.

Article published online March 29, 2008 most efficient use of experimentalist time by identifying processing windows for the formation of desired microstructures. This work presents a method by which processing conditions favorable for the formation of a desired microstructure may be systematically identified by using a combination of an analysis of nucleation and growth processes and phase field modeling. It is expected that, using approaches such as this, the process of microstructural design can be optimized by replacing the traditional approach of analysis, followed by experimentation, with analysis, followed by modeling, and then followed by experimentation.

Bimodal PSDs can develop through a variety of heat treatments. In the many superalloys, such as IN100, microstructure control is achieved by a series of step quenches, where the length and temperature of each isothermal hold controls the size and density of the particle populations. In another class of superalloys, such as Rene88DT, a simpler continuous cool process is employed. It is clear how a step quench could be used to produce a bimodal PSD, because nucleation rates can be controlled directly by the temperature of the hold. A more complex process is operative when bimodal PSDs are formed during continuous cooling. Previously, Gabb et $a l .{ }^{[2]}$ have shown that the bimodal PSD forms as a result of dynamic changes in nucleation rates that occur because of the interplay between nucleation and growth processes in a Langer-Schwartz, ${ }^{[3]}$ model and Wen et al. ${ }^{[4]}$ have shown that this can also occur in a phase field model. This work builds a framework, whereby heat treatment processes may be explored systematically through a combination of a zeroth order analysis of nucleation and growth phenomena that are used as a guide for phase field modeling. The zeroth order analysis uses simplified geometries and boundary conditions to make analytical predictions about the resultant 


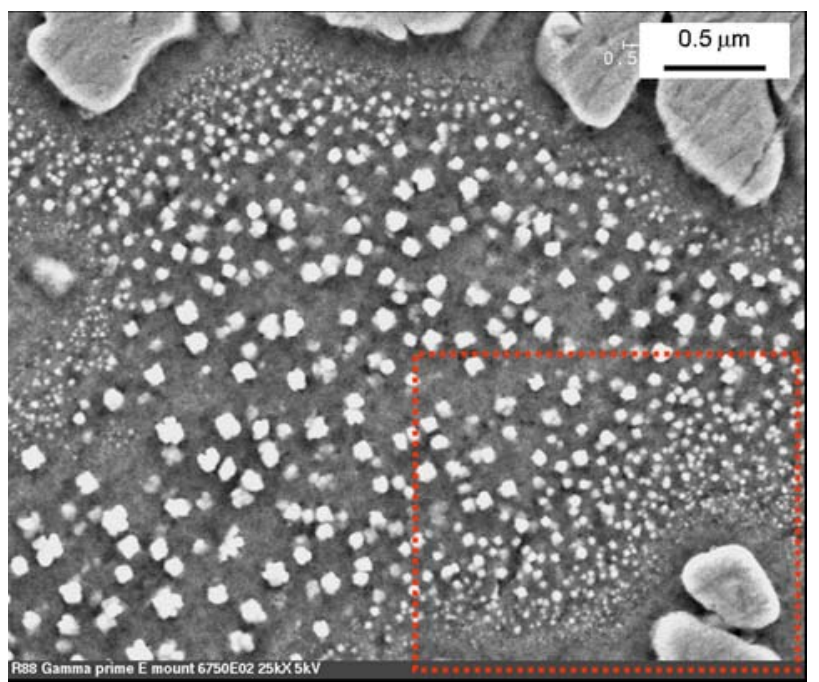

Fig. 1-SEM micrograph showing bimodal particle size distribution. Courtesy of M. Henry, G.E.

microstructure. Using these predictions as a guide, the heat treatments are refined further with the more accurate, but computationally intensive, phase field method. While we are interested in bimodal PSDs and the processing windows of their formation, this method is general and can be used for designing other microstructures that result from an interplay between nucleation and growth.

In supersolvus heat treatments, the alloy is heated to above the solvus and subsequently cooled. This can result in bimodal PSDs via a number of mechanisms. If aging above the supersolvus does not remove all the primary $\gamma$ particles, then subsequent cooling will produce a typical bimodal microstructure. Even if the supersolvus treatment removes all the primary $\gamma^{\prime}$ particles, the existence of heterogeneous nucleation sites may also yield a bimodal microstructure. The dynamic mechanism of bimodal PSD formation alluded to in the preceding discussion is distinct from these mechanisms, because it arises purely from the changes in the (homogeneous) nucleation rate.

The dynamic formation of bimodal PSDs is the result of the competition for supersaturation between nucleation of new particles and growth of existing ones. As existing particles grow, the supersaturation in the matrix diminishes, reducing the driving force for nucleation. As the temperature is lowered, the supersaturation of the matrix increases. The interplay among these two competing factors determines whether new particles will form and thus define a window for bimodal PSDs.

Bimodal PSD formation has been modeled, originally in Gabb et al., ${ }^{[2]}$ using a modification of the LangerSchwartz model ${ }^{[3]}$ that was applied toward the development of experimental alloy modifications of Rene88DT. Olson et al. ${ }^{[5]}$ used the commercial PrecipiCalc(tm) code that implements the Langer-Schwartz model for further investigations of bimodal PSD formation. Simulations were performed both under step quenching and continuous cooling. Wen et al. ${ }^{[4]}$ used a two-dimensional (2-D) phase field model that was nominally calibrated for binary Ni-Al growth and used uncalibrated parameters for nucleation and reported conditions for the formation of bimodal PSDs.

In Wen's work, conditions were identified under which a bimodal PSD would form under linear dimensionless continuous cooling conditions. Under these conditions, there is a steep drop in temperature at early times, which hinders the formation of bimodal PSDs. They identified three regions of behavior: (1) a monomodal (large) particle size distribution caused by a slow cooling, (2) a bimodal distribution caused by a moderate cooling rate, and (3) a mono-modal (small) distribution caused by a rapid cooling. This present study builds on the work of Wen et al. by (1) using an analysis of the nucleation and growth behavior that acts as a guide for phase field simulations to locate processing conditions favorable for the formation of bimodal PSDs, (2) expanding the range of cooling profiles to include a fully dimensional linear continuous cooling rate, and (3) incorporating the functional forms from classical nucleation theory $(\mathrm{CNT})^{[6]}$ in the phase field treatment of nucleation. Linear continuous cooling profiles were used in this article as a simple demonstration of this framework, but more complicated cooling profiles could be considered.

The explicit analysis of the nucleation and growth behavior confirmed that the mechanisms proposed by Wen et al. were actually responsible for the three regimes of PSD formation. Because phase field explicitly accounts for nonuniformities in composition of the untransformed matrix, it was found that this effect also aids in reactivation of nucleation at lower temperatures.

\section{CLASSICAL NUCLEATION THEORY}

The $\mathrm{CNT}^{[6]}$ was used in this article, both for the nucleation and growth analysis as well as for the nucleation treatment in the phase field. From CNT, the nucleation rate $N_{r}$ measures the change in the particle number density $\left(N_{v}\right)$ with time. In two dimensions,

$$
N_{r}=\frac{d N_{v}}{d t}=N_{0} \exp \left(\frac{-\Delta G^{*}}{\mathrm{k}_{\mathrm{b}} T}\right) \frac{D c}{\lambda^{2}} \frac{2 \pi r^{*}}{\lambda} \sqrt{\frac{\Delta G^{*}}{3 \pi \mathrm{k}_{\mathrm{b}} T}} \exp \left(\frac{-\tau}{t}\right)
$$

where $N_{0}$ is the number of atoms in the system; $\mathrm{k}_{\mathrm{b}}$, the Boltzmann constant; $T$, the temperature; $D$, the diffusivity; $c$, the concentration; $\lambda$, the nearest neighbor distance; $r^{*}$, the critical radius; and $\tau$, the incubation time. Under the assumption of steady-state nucleation, $\tau$ is 0 and that the population of subcritical clusters instantly achieves the proper distribution for any change in temperature and concentration. This is most applicable in the isothermal limit and for low cooling rates. At high cooling rates, this will tend to overrepresent the rate of nucleus formation.

The nucleation energy barrier $\Delta G^{*}$ is given by

$$
\Delta G^{*}=\frac{\pi \sigma^{2}}{\left(\Delta G_{s}+\Delta G_{c}\right)}
$$


The critical radius $r^{*}$ is

$$
r^{*}=\frac{\sigma}{\Delta G_{c}+\Delta G_{s}}
$$

where $\sigma$ is the interfacial energy, and $\Delta G_{s}$ and $\Delta G_{c}$ are respectively the coherency elastic strain energy and chemical free energy changes associated with the formation of the critical nucleus.

\section{NUCLEATION AND GROWTH MODELS}

The nucleation and growth analysis was developed from the nucleation and growth rates under idealized circumstances. Nucleation was modeled as steady-state nucleation within the CNT and was used as a predictor of the number of particles introduced per temperature increment. The growth rate was modeled by the growth of a diffusion-controlled particle in an infinite matrix and was used as a predictor for the solute depletion rate in solute per temperature increment. Contour plots of these growth and nucleation rates as functions of temperature and concentration were used to illustrate the behavior in the untransformed matrix.

\section{A. Nucleation Model}

The nucleation model indicates how the nucleation rate is affected by the supersaturation in the matrix. This is based on the CNT and is normalized by the cooling rate in order to account for the amount of time spent at each temperature. By the chain rule,

$$
n_{r}=\frac{d N_{v}}{d T}=\frac{d N_{v}}{d t} / \frac{d T}{d t}
$$

where $t$ is time, $T$ is temperature, and $\frac{d T}{d t}$ is the cooling rate. Thus, the expected change in $N_{v}$ per temperature step is given by

$$
\frac{d N_{v}}{d T}=N_{0} \exp \left(\frac{-\Delta G^{*}}{\mathrm{k}_{\mathrm{b}} T}\right) \frac{D c}{\lambda^{2}} \frac{2 \pi r^{*}}{\lambda} \sqrt{\frac{\Delta G^{*}}{3 \pi \mathrm{k}_{\mathrm{b}} T}} / \frac{d T}{d t}
$$

The nucleation rate has a peak at intermediate temperatures, and high concentrations, and produces the lower set of contour lines in Figure 2. This is a classical result that stems from the low driving force (supersaturation) at high temperatures and the low frequency factor (which varies with diffusivity) at low temperatures. For a given temperature, increasing the mean concentration at all temperatures increases the nucleation rate.

\section{B. Growth Model}

The growth model quantifies the amount of solute drained from the matrix as a result of particle growth. By mass balance, this is equal to the amount of solute added to the precipitate. As the cooling rate changes, the amount of time spent at each temperature changes, and thus the amount of solute depletion will change.

In a mean field model under nonisothermal conditions, similar to the expected number of nuclei calculation, the expected change in the concentration at each temperature is given by

$$
\frac{d \bar{c}}{d T}=\frac{d \bar{c}}{d t} / \frac{d T}{d t}
$$

where $\bar{c}$ is the mean concentration of untransformed matrix.

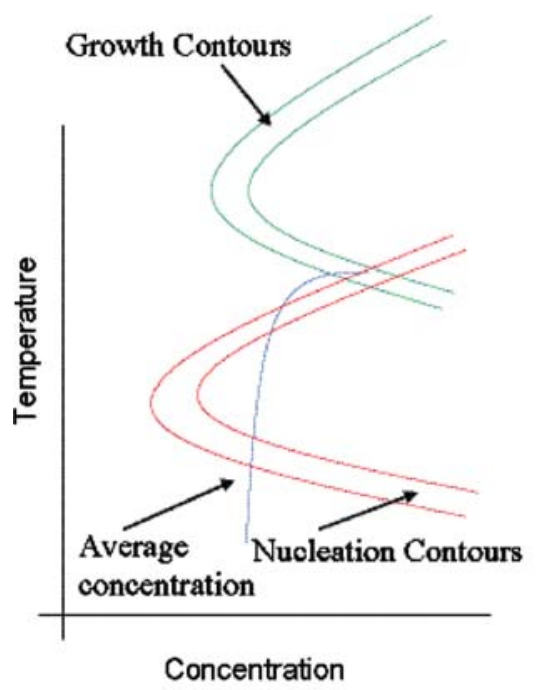

(a)

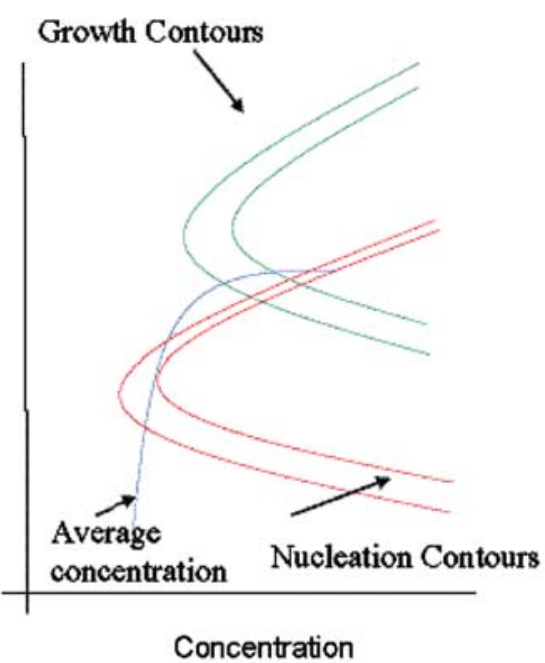

(b)

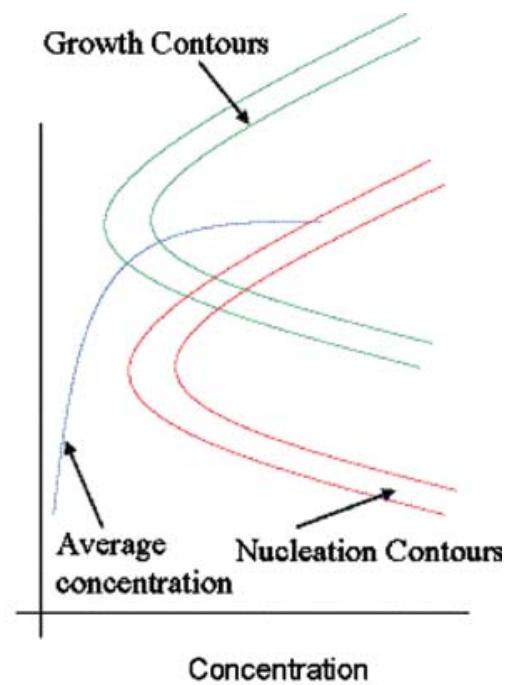

(c)

Fig. 2-Superposition of average concentration of the untransformed matrix onto contours of nucleation and growth under different cooling conditions and expected PSDs. (a) Fast cooling rates have more vertical concentration trajectories and form unimodal PSDs. (b) Bimodal PSDs form at moderate cooling rate. (c) Slow cooling rates have more horozontal trajectories and form unimodal PSDs. 
The value of $\bar{c}$ can be estimated from a mass balance, starting with a sharp interface model, ${ }^{[7]}$

$$
\left(A_{T}-\sum A\right)(\bar{c})+\sum A\left(c_{\gamma^{\prime}}\right)=A_{T}\left(c_{0}\right)
$$

where $A_{T}$ is the total area, $A$ is the precipitate area, $c_{\gamma}$ is the equilibrium concentration of the $\gamma$ phase, $c_{\gamma^{\prime}}$ is the equilibrium concentration of the $\gamma^{\prime}$ phase, and $c_{0}$ is the initial concentration, and the sum is taken over all particles.

Isolating $\bar{c}$ yields

$$
\bar{c}=\frac{A_{T} c_{0}}{A_{T}-\sum A}-\frac{\sum A c_{\gamma^{\prime}}}{A_{T}-\sum A}
$$

If the volume fraction is low, i.e., $A_{T} \gg \sum A$, then

$$
\bar{c}=c_{0}-\frac{\sum A c_{\gamma^{\prime}}}{A_{T}}
$$

and

$$
\frac{d \bar{c}}{d t}=-\frac{\sum A}{A_{T}} \frac{d c_{\gamma^{\prime}}}{d t}-\frac{c_{\gamma^{\prime}}}{A_{T}} \frac{d \sum A}{d t}
$$

According to the $\mathrm{Ni}-\mathrm{Al}$ phase diagram, ${ }^{[8]}$ for the temperature regime of interest, the variation of equilibrium $\gamma^{\prime}$ concentration with respect to temperature is small. Applying this assumption to Eq. [10] leads to

$$
\frac{d \bar{c}}{d t}=-\frac{c_{\gamma^{\prime}}}{A_{T}} \frac{d \sum A}{d t}
$$

Under the assumption of diffusion-controlled growth for $n$ two-dimensional (2-D) circular particles, the derivative for the sum in Eq. [11] can be replaced by $n \frac{d A}{d t}$, for the following reasoning. Under diffusion control the particle radius is described by

$$
r=\sqrt{2 \Omega D t}
$$

where $r$ is the particle radius. For circular particles, the area is given by

$$
A=\pi r^{2}=2 \pi \Omega D t
$$

where $\Omega$ is the supersaturation in the system given by

$$
\Omega=\frac{\bar{c}-c_{\gamma}}{c_{\gamma^{\prime}}-c_{\gamma}}
$$

Differentiating this,

$$
\frac{d A}{d t}=2 \pi \frac{\bar{c}-c_{\gamma}}{c_{\gamma^{\prime}}-c_{\gamma}} D+\frac{2 \pi D t}{c_{\gamma^{\prime}}-c_{\gamma}} \frac{d \bar{c}}{d t}
$$

From Eq. [15], the first term is time and particle independent, so for $n$ particles,

$$
\frac{d \sum A}{d t}=\sum \frac{d A}{d t}=n 2 \pi \frac{\bar{c}-c_{\gamma}}{c_{\gamma^{\prime}}-c_{\gamma}} D+\sum \frac{2 \pi D t}{c_{\gamma^{\prime}}-c_{\gamma}} \frac{d \bar{c}}{d t}
$$

Combining Eq. [11] and Eq. [16] gives

$$
\frac{d \bar{c}}{d t}=-2 \pi n \frac{c_{\gamma^{\prime}}}{A_{T}} \frac{\bar{c}-c_{\gamma}}{c_{\gamma^{\prime}}-c_{\gamma}} D /\left(1+\frac{c_{\gamma^{\prime}}}{A_{T}} \sum \frac{2 \pi D t}{c \gamma^{\prime}-c \gamma}\right)
$$

where for slow diffusing species, the second term in the denomoninator is clearly much less than 1 , so that

$$
\frac{d \bar{c}}{d t}=-2 \pi n \frac{c_{\gamma^{\prime}}}{A_{T}} \frac{\bar{c}-c_{\gamma}}{c_{\gamma^{\prime}}-c_{\gamma}} D
$$

Substituting Eq. [18] into Eq. [6], the cooling rate normalized depletion rate (NDR) is

$$
-\frac{d \bar{c}}{d T}=2 \pi n \frac{c_{\gamma^{\prime}}}{A_{T}} \frac{\bar{c}-c_{\gamma}}{c_{\gamma^{\prime}}-c_{\gamma}} D / \frac{d T}{d t}
$$

When the NDR is plotted against concentration and temperature axes, it produces the upper set of contours in Figure 2. The normalization produces the amount of solute depletion expected per degree temperature drop and implicitly accounts for the amount of time the system spends at each temperature, i.e., cooling rate.

The NDR is multivalued and has a peak at intermediate temperatures. At high temperatures, diffusion is fast, but supersaturation is low. At low temperatures, supersaturation is high, but diffusivity is low. At a given temperature, an increased supersaturation results in an increased NDR.

\section{Contour Plot Use}

The growth and nucleation contours were plotted together to illustrate how their interaction predicts the eventual microstructure (Figure 2). In slow cooling (Figure 2(c)), the system spends a lot of time at each temperature, so the NDR due to growth is greater than the supersaturation augmentation due to undercooling. This suppresses nucleation so a unimodal PSD is expected. In fast cooling situations, the system spends little time at each temperature, so the increase in supersaturation due to undercooling is greater than the depletion due to growth, causing continuous nucleation. In order to form bimodal PSDs, the NDR must be initially fast to shut down nucleation, but then drop off to allow undercooling to augment the supersaturation, producing a second burst of nucleation.

In general, faster cooling contour curves are shifted to the right and separated. If the curves are close together, then the microstructure will be a unimodal distribution of large particles. If the curves are far apart, then the microstructure will be a unimodal distribution of small particles. A moderate cooling rate promotes a bimodal PSD, which can be seen as a slow cool too fast to deplete all the available saturation, or a fast cool too slow to completely suppress growth.

\section{MICROSTRUCTURE MODELING}

The phase field modeling in this study was based on previous work on the $\mathrm{Ni}-\mathrm{Al}$ system. ${ }^{[4,9,10]}$ The principal 
extensions made in the current study are (1) the incorporation of CNT to account for the temperature dependence of the nucleation treatment and (2) consideration of cooling in dimensional space.

\section{A. Phase Field Method}

In the phase field methodology, the microstructural evolution of a material system is described by the evolution in composition $(c(\mathbf{r}, t))$ and long-range ordering $\left(\eta_{i}(\mathbf{r}, t)\right)$ field variables. The Osanger type CahnHilliard ${ }^{[11]}$ and Ginzburg-Landau ${ }^{[12]}$ equations were used in conjunction with the CNT to simulate particle evolution. The parameters for the Cahn-Hilliard and Ginzburg-Landau equations were chosen to represent the NiAl material system based on previous simulation work. ${ }^{[9]}$

The evolution of the system is described by

$$
\frac{\partial c(r, t)}{\partial t}=\nabla\left(M \nabla \frac{\partial F}{\partial c(r, t)}\right)
$$

and

$$
\frac{\partial \eta_{i}(r, t)}{\partial t}=-L \frac{\partial F}{\partial \eta_{i}(r, t)}
$$

where these field variables vary in spacial position $(r)$ and time $(t)$.

In Eqs. [20] and [21], $F$ is the total free energy, $M$ the Chemical mobility, and $L$ the kinetic coefficient for longrange ordering parameter.

The total free energy is described by

$$
F=\int_{V}\left[\frac{k_{c}}{2}(\nabla c)^{2}+\frac{k_{\eta}}{2} \sum_{i}\left(\nabla \eta_{i}\right)^{2}+f\left(c, \eta_{i}\right)\right] d V
$$

where $f$ is the local free energy density and $k_{c}$ and $k_{\eta}$ are the gradient energy coefficients. The coherency elastic energy is ignored because of the small lattice misfit in disk alloys.

The governing equations were normalized to a nondimensional form for numerical considerations

$$
\frac{\partial c(r, t)}{\partial t}=\chi \nabla^{* 2}\left(-k_{c}^{*} \nabla^{* 2} c+\frac{\partial f^{*}}{\partial c(r, t)}\right)
$$

and

$$
\frac{\partial \eta_{i}(r, t)}{\partial \tau}=k_{\eta}^{*} \nabla^{* 2} \eta_{i}-\frac{\partial f^{*}}{\partial \eta_{i}(r, t)}
$$

where

$$
\begin{gathered}
\chi=\frac{M}{L l_{0}^{2}} \\
k_{c}^{*}=\frac{k_{c}}{\Theta_{c h} l_{0}^{2}} \\
k_{\eta}^{*}=\frac{k_{\eta}}{\Theta_{c h} l_{0}^{2}}
\end{gathered}
$$

$$
f^{*}=\frac{f}{\Theta_{c h}}
$$

and

$$
\tau=L \Theta_{c h} t
$$

where $l_{0}$ is the length of a simulation grid and $\Theta_{c h}$ is a typical normalization factor of the free energy.

The parameters used in this study are based on the ones used in Wen et al. ${ }^{[9]}$ and are listed in Table I.

\section{B. Poisson Seeding}

New particles were introduced into the simulations based on the model developed by Simmons et al.$^{[10]}$ This model was modified by incorporating the $\mathrm{CNT}^{[6]}$ and the critical nucleus radius.

According to, ${ }^{[6]}$ the probability of nucleation is given as

$$
P_{\text {nucleation }}=1-\exp \left(N_{r} * \Delta t\right)
$$

The nucleation rate was reduced to dimensionless form by using Eqs. [25] and [29], along with

$$
D=M \frac{d^{2} f}{d c^{2}}
$$

for converting the diffusivity to the Einstein mobility, ${ }^{[13]}$ and

$$
N_{0}=l_{0}^{2}
$$

for converting the nucleation site density to grid units. Making this substitution, Eq. [30] becomes

$$
P_{\text {nucleation }}=1-\exp \left(N_{r}^{*} \Delta \tau\right)
$$

where

$$
N_{r}^{*}=l_{0}^{2} \exp \left(\frac{-\Delta G^{*}}{\mathrm{k}_{\mathrm{b}} T}\right) \frac{\chi \frac{d^{2} f}{d c^{2}} c}{\lambda^{2}} \frac{2 \pi r^{*}}{\lambda} \sqrt{\frac{\Delta G^{*}}{3 \pi \mathrm{k}_{\mathrm{b}} T}} / \Theta_{c h}
$$

Supercritically sized particles were used to ensure survivable particles. In practice, this was accomplished by multiplying the critical radius by a constant $(A)$, which both converted to dimensionless form and

Table I. Parameters Used in Simulations

\begin{tabular}{llr}
\hline Parameter & \multicolumn{1}{c}{ Value } & Reference \\
\hline$L$ & $1.0 \times 10^{6} \exp (-13,118.3 / T)$ & 4 \\
$f$ & normalized Ansara 97 & 16 \\
$\sigma$ & $14 \mathrm{~mJ} / \mathrm{m}$ & 17 \\
$\chi$ & 0.05 & 4 \\
$\kappa_{c}$ & 45 & 4 \\
$\kappa_{\eta}$ & 0 & 4 \\
$\theta$ & 500 & 4 \\
$l_{0}$ & $0.5 \mathrm{~A}$ & 4 \\
\hline
\end{tabular}


increased the seed size to a survivable one. Typically, $A=3 / l_{0}$ was used in this work.

$$
r_{\text {dimless }}^{*}=A * r_{\text {dimensional }}^{*}
$$

\section{Dimensional vs Dimensionless Cooling Profiles}

In Wen et al.,${ }^{[4]}$ the simulations were performed with a linear dimensionless cooling rate. When converting this to a dimensional cooling rate, the dimensionless time is rescaled to the dimensional time by Eq. [29]. This requires that the temperature increment be scaled (with absolute temperature) to maintain a constant isothermal integration time, the isothermal integration time be scaled to maintain a constant temperature increment, or some combination of both. In this work, a constant temperature increment was applied and the phase field equations were integrated over a temperature-dependent variable isothermal time.

The temperature time profile for $6{ }^{\circ} \mathrm{C} / \mathrm{s}$ is shown schematically in Figure 3. The linear cooling rate in real time is approximated by a series of isothermal steps, each 1 deg apart. These isothermal steps are then converted to dimensionless time using Eq. [29].

\section{Simulation Parameters}

The simulations were designed to produce bimodal microstructures under linear cooling rates. For the initial concentrations chosen, the simulations ran from the supersolvus temperature until $400{ }^{\circ} \mathrm{C}$, when significant nucleation and growth were both shut off due to the low diffusion rate. Dimensional cooling rates of
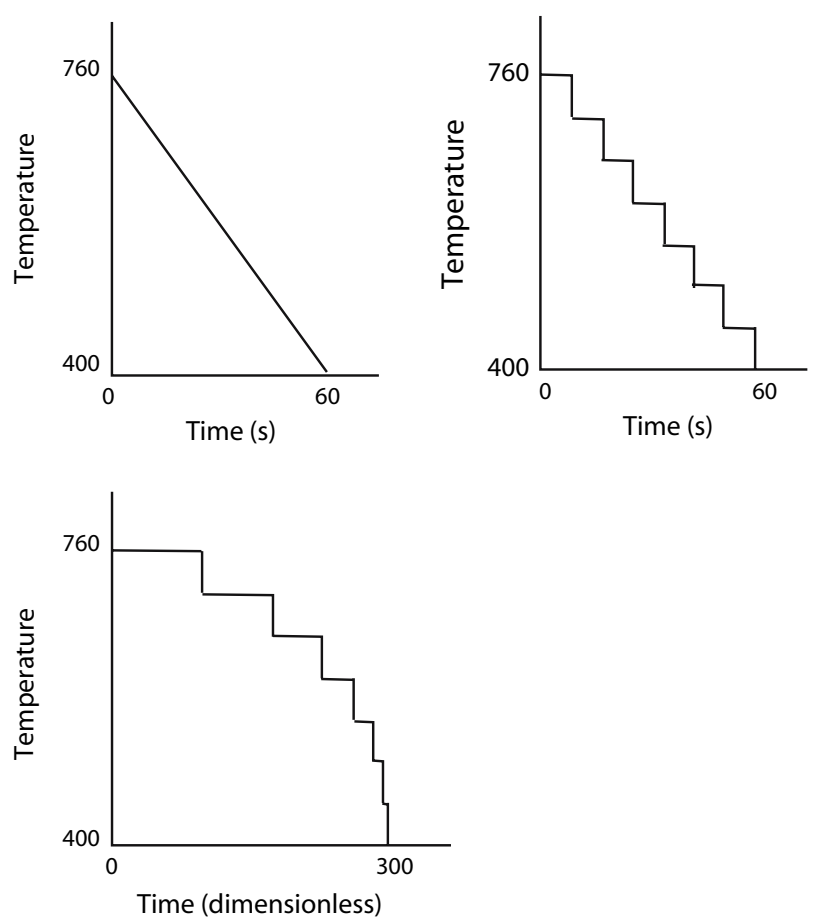

Fig. 3-Comparison of dimensional and dimensionless cooling curves.
$100{ }^{\circ} \mathrm{C} / \mathrm{s}, 50{ }^{\circ} \mathrm{C} / \mathrm{s}, 25^{\circ} \mathrm{C} / \mathrm{s}$, and $5{ }^{\circ} \mathrm{C} / \mathrm{s}$ were simulated. The simulations were 2-D, with 1024 grid points in each direction and periodic boundaries.

Numerical integration of Eqs. [23] and [24] was carried out under specific time and temperature profiles. The Runge-Kutta 4-5 adaptive method was used in order to maximize the time-steps allowable, while controlling the estimated error introduced with each time-step.

Because higher temperatures require more dimensionless time to simulate, the concentration of $\mathrm{Al}$ was set to 13.9 in order to lower the solvus temperature. Initial testing showed that for this concentration, homogeneous nucleation began around $760{ }^{\circ} \mathrm{C}$.

\section{RESULTS}

The three fast cooling rates produced a continuous range of PSDs from unimodal (small) towards bimodal. The slowest cooling rate $\left(5^{\circ} \mathrm{C} / \mathrm{s}\right)$ produced a bimodal PSD. These are shown in Figures 4 and 6, respectively. The results of the analytical model are shown in Figures 5 and 7, respectively.

In Figures 5 and 7, the trajectories of the mean and maximum concentrations of the untransformed matrix are plotted against the growth and nucleation contours. In addition, the temperature and composition of each nucleation event is marked with an $\mathrm{x}$. The maximum untransformed concentration bounds the x's to the right, while the mean transformation bounds the nucleation events to the left. Regions of high normalized depletion rate cause the trajectories to flatten out, indicating more supersaturation depletion per temperature step. Regions of high nucleation are predicted when the trajectories are within the nucleation contours and are evidenced by x's.

The microstructure of the $100{ }^{\circ} \mathrm{C} / \mathrm{s}$ simulation is shown in Figure 4(a) and its corresponding analytical model in Figure 5(a). The aggressive cooling rate continually introduced new particles into the system, and while mass conservation dictated that the average concentration of untransformed matrix decreased, the maximum concentrations of untransformed matrix (far away from the particles) remained unchanged. This implies that the particles are not growing much and do not have time to extend their diffusion fields.

The microstructures for the $50{ }^{\circ} \mathrm{C} / \mathrm{s}$ and $25^{\circ} \mathrm{C} / \mathrm{s}$ simulations and their respective contour plots can be seen in Figures 4(b) and (c) and Figures 5(b) and (c). The microstructures and analytical models showed how existing particles began to establish soft impingement regions and retard subsequent nucleation. The development of a small gap around $720{ }^{\circ} \mathrm{C}$ in Figure 5(c) during which no nuclei are introduced indicated the onset of a bimodal PSD.

The microstructural evolution obtained for the case of the $5{ }^{\circ} \mathrm{C} / \mathrm{s}$ cooling is shown in Figure 6. The figure shows the intermediate steps the microstructure went through. After the initial period of nucleation, concentration trajectory (Figure 7) showed that the maximum concentration rapidly diminishes. This implied that the 


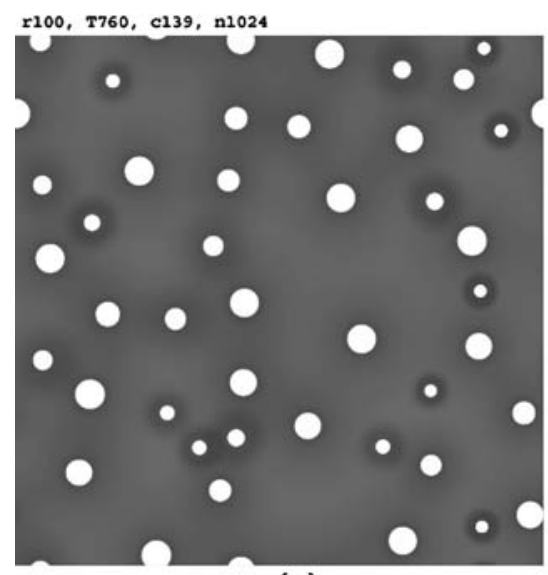

(a) r50, c139, Tinit 760, n1024

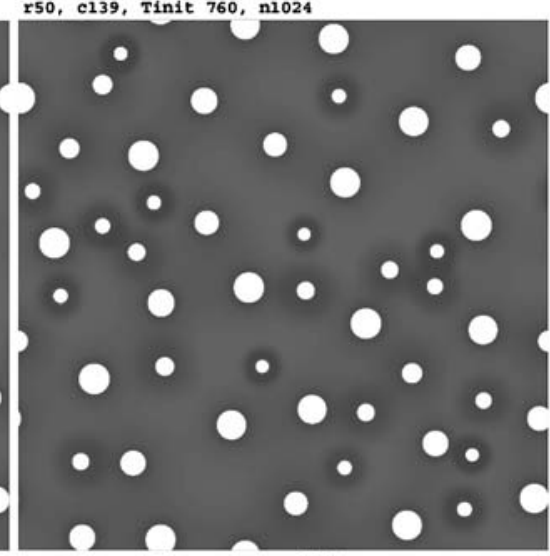

(b)

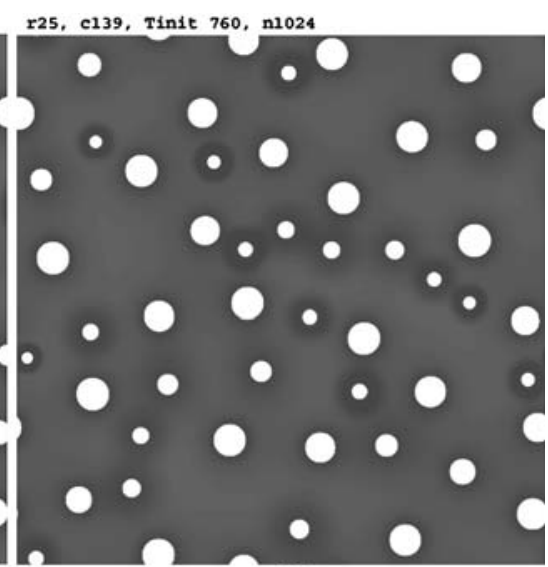

(c)

Fig. 4 - Final simulated microstructures under different cooling rates. (a) $100{ }^{\circ} \mathrm{C} / \mathrm{s},(b) 50{ }^{\circ} \mathrm{C} / \mathrm{s}$, and $(c) 25^{\circ} \mathrm{C} / \mathrm{s}$.

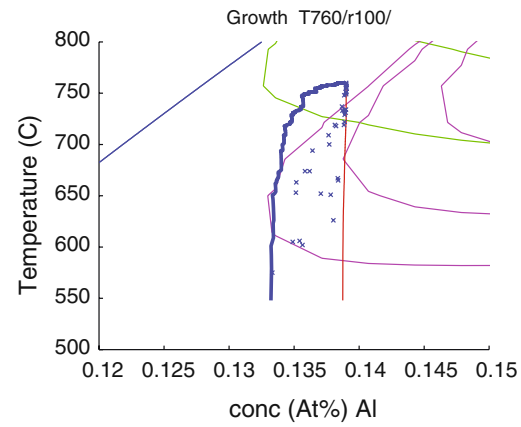

(a)

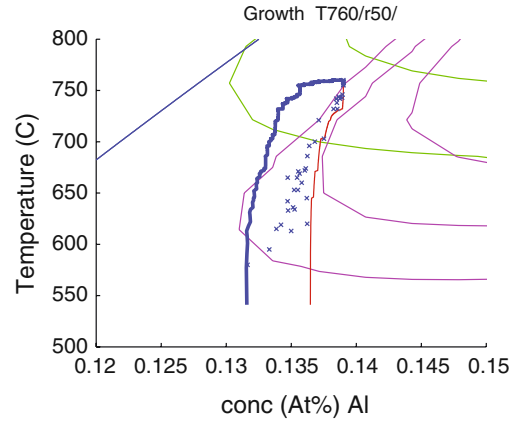

(b)

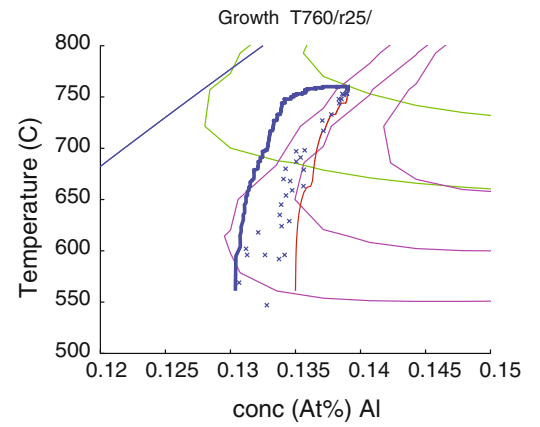

(c)

Fig. 5- Superposition of average (blue line) and maximum (red line) onto nucleation (purple lines) and growth (green lines) contours for different cooling rates. (a) $100{ }^{\circ} \mathrm{C} / \mathrm{s}$, (b) $50{ }^{\circ} \mathrm{C} / \mathrm{s}$, and $(c) 25^{\circ} \mathrm{C} / \mathrm{s}$. The x's indicate individual nucleation events.

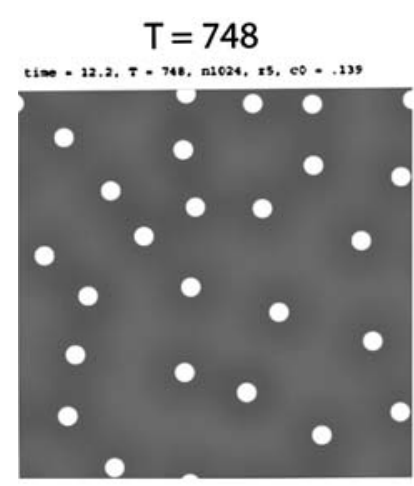

$T=683$

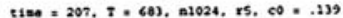

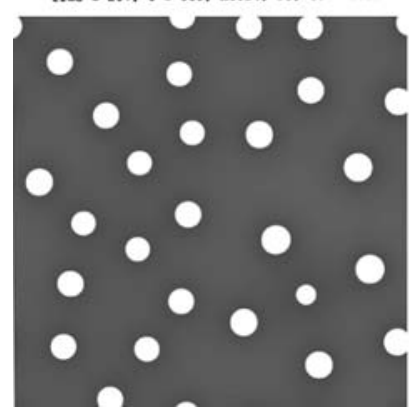

$$
T=746
$$

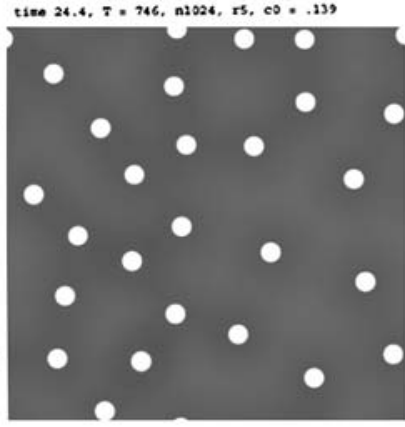

$$
T=670
$$

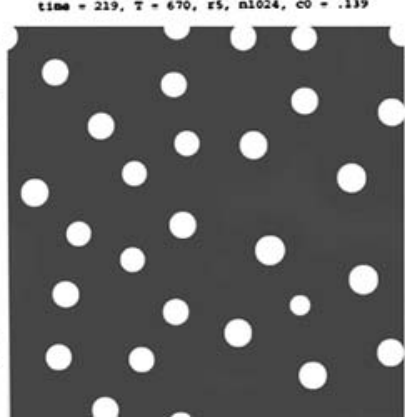

$\mathrm{T}=744$

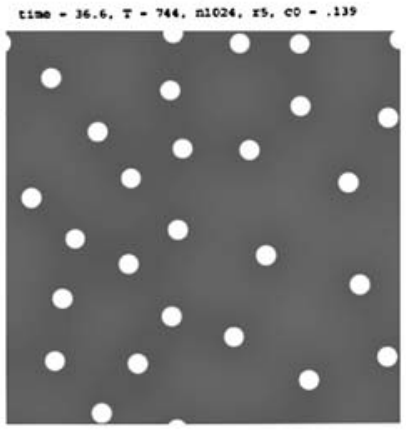

$T=650$

$0.232,=-643,55,02024,60-139$

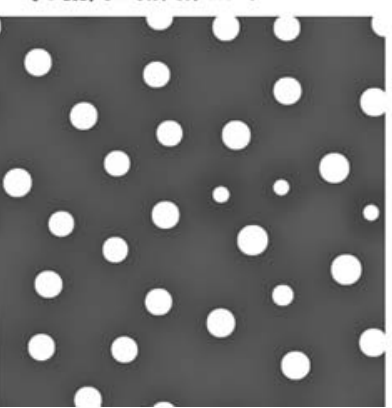

$\mathrm{T}=700$

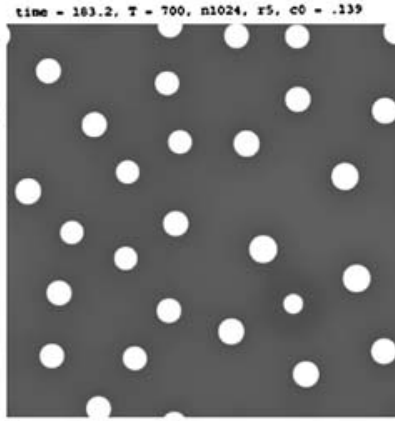

$\mathrm{T}=447$

Fig. 6-Formation of a bimodal microstructure with a cooling rate of $5{ }^{\circ} \mathrm{C} / \mathrm{s}$. 
existing particles have overlapping diffusion fields to prevent new particles from nucleating. Nucleation was not resumed until $650{ }^{\circ} \mathrm{C}$ when the cooling has built up enough supersaturation in the matrix phase. This leads to a typical bimodal microstructure.

\section{DISCUSSION}

The phase field model, coupled with the nucleation and growth analysis presented here, is a useful tool for designing microstructures of desired types. In particular, this article focused on developing bimodal microstructures, which form by dynamic nucleation behavior. In this case, the nucleation rate varies dynamically, even during a straight line cool, to produce the bimodal microstructure. This phenomenon was originally found to exist with a Langer-Schwartz model by Gabb et al. ${ }^{[2]}$ and shown to also exist in the phase field model by Wen et al. ${ }^{[4]}$ This work gives a systematic methodology for designing microstructures of this type by a simple analysis of the competition between existing and potential particles in the microstructure coupled with the phase field model for treatment of nonuniform effects.

Here, the competition between existing and potential particles is examined with both the analytical model and phase field in order to identify conditions for formation of a bimodal PSD. By using the simpler analytical model to guide the computationally more expensive phase field model, a processing window was determined, within which the bimodal microstructure is expected to form. The 2-D phase field model accounts for the interacting diffusion fields and could be used to account for elastic interactions, both of which are absent in the analytical model. Although this work does not incorporate elastic interactions, which is specific to the material system considered. ${ }^{[14,15]}$

This work gives a quantitative explanation for the three limiting cases of the transformation behavior, as presented by Wen et al., ${ }^{[4]}$ i.e., (1) a soft-impingement regime, where nucleation is stopped by soft impingement and never start again; (2) a bimodal microstructure regime, where nucleation restarts at a lower temperature due to supersaturation build-up; and (3) a continuous nucleation regime, where the supersaturation is always sufficient to support nucleation. Here, the analysis of the nucleation and growth rates shows that bimodal PSDs can form by the dynamic interplay between these two effects. By direct computation of the nucleation and growth rates, these mechanisms for microstructural development were shown to be controllable.

In dynamically formed bimodal PSDs, the system has two peaks in the nucleation rate, as the system cools, as shown in Figure 8, where the dimensionless nucleation rates are plotted as a function of dimensionless time. The first peak is when the secondary particles form, while the second peak represents the formation of the tertiary particles. These correspond to the concentration trajectory bending to reintersect the nucleation contour due to supersaturation buildup.
The current work improves on the work by Wen et al. ${ }^{[4]}$ by finding a processing window for linear dimensional cooling rates. Wen et al. found dynamic bimodal PSD formation during a linear dimensionless cooling rate, which is generally a curved time-temperature profile in dimensional time. The dimensionless scaling factor between dimensional and dimensionless time varies as temperature changes (Eq. [29]).

With the method used here, the temperature was incremented at a constant rate, which means that the (dimensional) time for evolution at that temperature increased with decreasing temperature. When the real time linear cooling curve is converted into dimensionless time, it resembles an isothermal hold followed by a rapid quench (Figure 3), facilitating the task of locating conditions under which bimodal PSDs form. While linear dimensional cooling rates were chosen for study here, more complex cooling profiles are also possible and are being investigated presently.

A more general approach to the problem of microstructure design is illustrated in Figure 9, which shows the nucleation and growth analysis as a simple front end to the phase field method to explore conditions favorable for the formation of target microstructures in advance of experimentation. The nucleation and growth analysis is a very fast-acting (real time) method that can be used to target the slower 2-D phase field method, which must be iterated to find a solution.

The ability to treat microstructural inhomogeneity is often given as an advantage of the phase field method over mean field models. Figure 6 shows that this inhomogeneity indeed plays an important role in the formation of bimodal microstructures. Nucleation events can be seen to favor sites near the maximum available concentration. Because the nucleation rate is a strong function of the supersaturation, local enhancements in solute in the untransformed matrix are expected to be sites for nucleation of tertiary particles. This would give rise to the realistic microstructures shown in Figure 1.

The microstructures given in Figure 6 do not show the clustering of the tertiary particles that would be expected. This is due to computational limitations, not limitations in the phase field model per se. Because solving the phase field equations requires that the system be discretized, there are generally a minimum of about 100 grid points required to represent a single particle. That is, a minimum of three grid points will be required for each feature to be accurately represented. A particle has a boundary on each side and the uniform region in between, and the channel between it and neighboring particles, leading to a minimum diameter of approximately 12 grid points per particle. This gives approximately 100 grid points needed for the entire area. The particle number densities in Figure 1 were not simulated in this work, due to the large quantities of computer time required.

This process can be made more efficient by insertion of a Langer-Schwartz model between the nucleation and growth analysis and the 2-D phase field modeling as well as by the insertion of three-dimensional phase field modeling between the 2-D phase field modeling and 


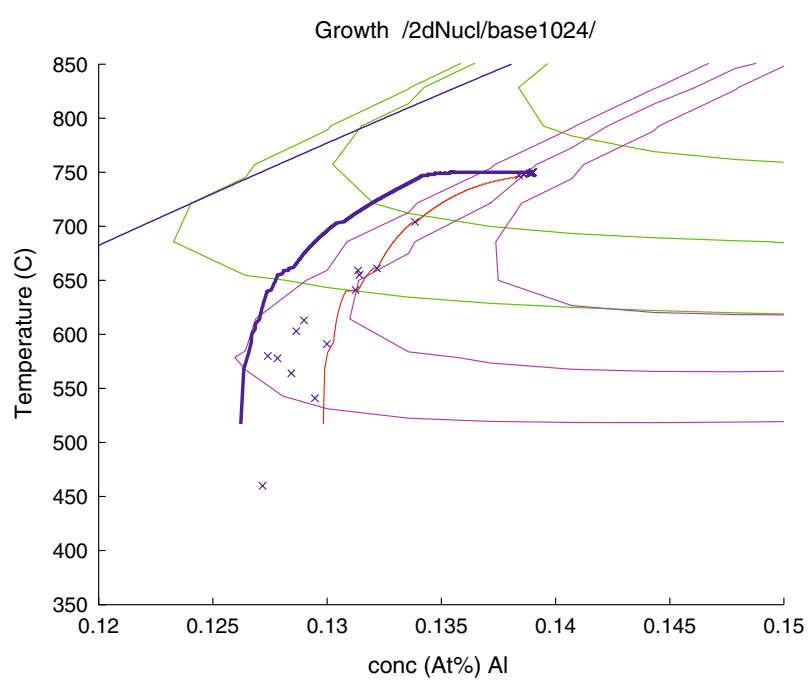

Fig. 7-Bimodal microstructure analysis of a $5{ }^{\circ} \mathrm{C} / \mathrm{s}$ cooling rate.

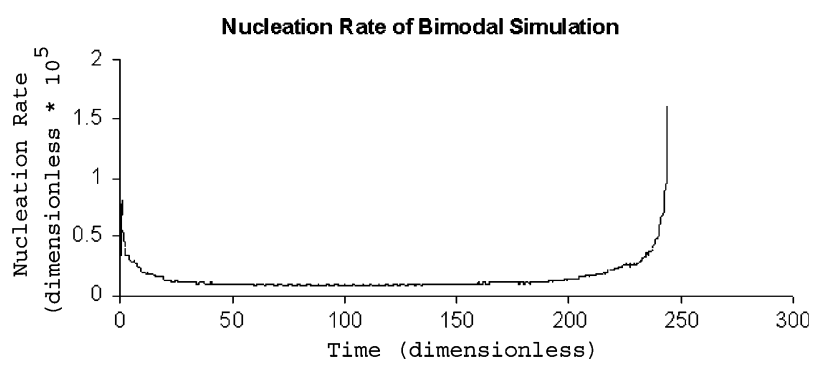

Fig. 8-Nucleation rate under the $5{ }^{\circ} \mathrm{C} / \mathrm{s}$ cooling rate.

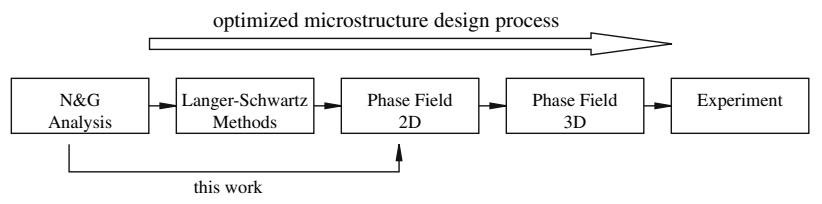

Fig. 9-Model-optimized process for microstructural design.

experiment to achieve more quantitative predictions of the kinetics. In particular, the particle number density, particle spacing, and growth kinetics are expected to be strongly influenced by dimensionality.

The methods shown in Figure 9 are arranged in the order of the most inexpensive to the most expensive so that an optimal reduction of the risks may be achieved, with each successive technique providing additional information. The nucleation and growth analysis gives an estimate of the nucleation behavior in the untransformed regions. This would be improved by the LangerSchwartz model, which would relax a simplifying assumption of uniform particle size to produce dynamically evolving particle size distributions. The 2-D phase field model improves on this by adding the effects of nonuniformity in the untransformed matrix composition. In particular, the phase field method accounts for extreme values in untransformed matrix composition. Because the supersaturation is the greatest in the channels between the secondary particles, tertiary particles tend to form there, as can be seen in Figure 1. This can be seen by the nucleation events tending toward the maximum untransformed concentration trajectories in Figures 5 and 7 at higher temperatures, until undercooling caused by the rapid cooling allows more sites to be viable nucleation locations.

\section{SUMMARY}

Continuous cooling simulations of a model Ni-Al system were performed at various rates, and a bimodal PSD was systematically found. A mean field model was used to help explain and guide each iteration. Together, these two models are used as a framework for designing microstructures by linking analytical models with the phase field methodology in order to narrow the window of heat treatments, which needs to be explored experiementally to find microstructures with particular characteristics.

\section{ACKNOWLEDGMENTS}

The authors express appreciation to Drs. Mike Mills and Peter Sarosi for their helpful discussions. Computations were performed on the SGI Altix 3700 at the ASC Major Shared Resource Center at Wright-Patterson AFB. One of the authors (YHW) acknowledges support from the Air Force Research Laboratory through Contract No. FA8650-04-D-5233 with UES Inc. Two of the authors (BW and YW) acknowledge support from the AFRL under the STW-21 program through Contract No. F33615-01-2-5225. All technical content contained herein was released under Case No. AFRL/W5 07-0105.

\section{REFERENCES}

1. G. Viswanathan, P. Sarosi, M. Henry, D. Whitis, W. Milligan, and M.J. Mills: Acta Mater., 2005, vol. 53, pp. 3041-57.

2. T. Gabb, D.G. Backman, D.Y. Wei, D.P. Mourer, D. Furrer, A. Garg, and D. Ellis: SuperAlloys 2000, TMS, Warrendale, PA, 2000, pp. 405-14.

3. J. Langer and A. Shwartz: Phys. Rev., 1980, vol. A21, pp. 948-58.

4. Y. Wen, J. Simmons, C. Shen, C. Woodward, and Y. Wang: Acta Mater., 2003, vol. 51, pp. 1123-32.

5. G. Olson: Sci., 2000, vol. 288, pp. 993-98.

6. H. Aaronson: Lecture on the Theory of Phase Transformations, TMS-AIME, Warrendale, PA, 1975, pp. 83-111.

7. R. Doherty: in Physical Metallurgy, 4th ed., R. Cahn and P. Hassen, eds., North Holland, Amsterdam, 1996, vol. 2, pp. 1364 505 .

8. W. Huang and Y. Chang: Intermetallics, 1998, vol. 6, pp. 487-98.

9. Y. Wen, B. Wang, J. Simmons, and Y. Wang: Acta Mater., 2006, vol. 54, pp. 2087-99.

10. J. Simmons, C. Shen, and Y. Wang: Scripta Mater., 2000, vol. 43, pp. $935-42$.

11. J. Cahn: J. Chem. Phys., 1958, vol. 28, pp. 258-67.

12. J. Gunton, M. Miguel, and P. Sahni: in Phase Transitions and Critical Phenomena, C. Domb and J. Lebowitz, eds., Academic Press, New York, NY, 1983, pp. 267-466. 
13. J. Cahn: TMS-AIME, 1968, vol. 242, pp. 166-80.

14. C. Shen, J. Simmons, and Y. Wang: Acta Mater., 2006, vol. 54, pp. 5617-30.

15. C. Shen, J. Simmons, and Y. Wang: Acta Mater., 2007, vol. 55, pp. 1457-66.
16. I. Ansara, N. Dupin, H. Lukas, and B. Sundman: J. Alloy Compd., 1997, vol. 247, pp. 20-30.

17. A. Ardell: Acta Metall., 1968, vol. 16, pp. 511-16. 